\title{
Characterizing Species at Risk I: Modeling Rare Species Under the Northwest Forest Plan
}

\author{
Bruce G. Marcot $^{1}$
}

\begin{abstract}
The Northwest Forest Plan in the Pacific Northwest, United States includes directives for survey and site protection of hundreds of rare species across many taxonomic classes. To help direct survey activities, prioritize sites, and stand conditions for conservation of these species, I developed Bayesian belief network $(\mathrm{BBN})$ models of habitat relationships and multiple stressors predicting presence of 12 rare species, and I present an example of predicting presence and absence of a rare fungus. The BBN models are developed along a rigorous process of expert judgment, peer review, reconciliation, accuracy testing, and incremental updating with known site data and validation data. Management implications of prediction errors are discussed.
\end{abstract}

Key Words: Bayesian belief networks; Bridgeoporus nobilissimus; model validation; Northwest Forest Plan; rare species models; Pacific Northwest.

\section{INTRODUCTION}

The Northwest Forest Plan (NWFP) is an ambitious regional land management plan instituted in 1994 to guide conservation of old-forest biodiversity on federal public lands in the Pacific Northwest United States (USDA and USDI 1994). One of the aims of the NWFP is to ensure persistence of species closely associated with late-successional and old-growth (LSOG) forests.

To aid this objective, as part of the NWFP program, a series of models were built to predict suitability of sites for, and presence of, rare and little-known LSOG-associated species. The models were built at two spatial scales: a broad ecoprovince scale, and a site-specific scale. The broadscale models were built in a geographic information system (GIS) to predict potential environmental suitability of selected species based on climatic and geophysical variables and potential natural vegetation across landscapes (J. Henderson, personal communication; Lesher 2005). I led the effort to develop site-specific models using Bayesian belief networks (BBNs). The site-specific models could be run independent of the broadscale models. Alternatively, one could use the broadscale models first to map large polygon areas of potentially suitable environments for a species, and then apply the site-specific models within those polygons to refine the predictions, because many of the species modeled respond as much to very fine grained environmental and habitat features that are not represented in GIS, as they do to broader-scale features. To date, the site-specific models are of 12 rare and little-known LSOG species, i.e., two fungi, three lichens, one moss, two vascular plants, two mollusks, one amphibian, one mammal. I present the BBN modeling work in this paper using one of the fungus models as an example. This paper explains how BBNs can be used to produce ecological prediction models; a companion paper (Marcot et al. 2006) explains use of BBNs for producing decision-aiding models also under the NWFP.

\section{METHODS}

\section{What is a Bayesian belief network?}

A Bayesian belief network (BBN) is essentially a set of variables, represented as a network of nodes that are linked by probabilities. The nodes represent correlates that affect some outcome(s) of interest, and the links represent how the correlates interact, that is, the influence among the nodes (Marcot et al. 
2001). At its simplest, a BBN is what is termed an "influence diagram," which is a figure of nodes, $i$. e., variables, and arrows, i.e., links, with an underlying probability structure. Nodes without incoming arrows are predictor variables, nodes without outgoing arrows are response variables, and nodes with both incoming and outgoing arrows are latent or calculated variables. Formally, a BBN is an acyclic graph, that is, a network with no feedback loops, where ideally the predictor variables are not just correlates but are direct or indirect causes of response variables.

Throughout this paper, I will use one example species for which I developed, tested, and updated BBN models in conjunction with species experts. I used the BBN modeling software Netica (Norsys, Inc., http://www.norsys.com). These models predict presence and absence of a rare species of fungus called fuzzy sandozi, Bridgeoporus [prev. Oxyporus] nobilissimus (Basidiomycota, Polyporaceae, Aphyllophorales) (Burdsall etal. 1996). This fungus forms large hard conks, some of the largest fungus fruiting bodies in the world, on the base of largediameter live boles, snags, and stumps mostly of noble fir (Abies procera) in mature, mesic forests of the Pacific Northwest (T. O'Dell and T. Dreisbach, personal communication). This paper is not intended to be a definitive analysis of this species, but rather an exposition of the $\mathrm{BBN}$ modeling process of a rare species managed under the NWFP. BBN models, for 11 other species under NWFP are in various stages of completion.

\section{Use of Bayesian belief networks for species- habitat modeling}

In recent years, BBNs have become a popular means of modeling species-habitat and stressor relationships. Rowland et al. (2003) used BBNs to model landscape use by wolverine (Gulo gulo) in the interior west United States. Schnute et al. (2000) used BBN analysis to establish goals for managing Fraser River sockeye salmon (Oncorhynchus nerka). Lee (2000) used BBNs to model land-use effects on bull trout (Salvelinus confluentus). Many other examples are available (e.g., Raphael et al. 2001, Lehmkuhl et al. 2001).

\section{Probability structure of a Bayesian belief network model}

BBNs are probabilistic models that depict the frequencies or prior probabilities of various states of input, i.e., predictor variables, the conditional probabilities of intermediate variables, and the posterior probabilities of various states of output, $i$. e., response variables (Jensen 1996). In brief, the statistical structure of a BBN is as follows (Spiegelhalter et al. 1993).

In BBNs, prior probabilities of input variables are traditionally represented as being distributed as a discontinuous Dirichlet function

$$
D(x)=\lim _{m \rightarrow \infty} \lim _{n \rightarrow \infty} \cos ^{2 n}(m ! \pi x)
$$

(Weisstein 2005), which is a multivariate generalization of the two-state Beta distribution (Marin et al. 2003) in which state probabilities are continuous and bounded in the interval $[0,1]$ (Castillo et al. 1997). Conditional probabilities are presumed independent of one another and are specified either by the modeler or are calculated from case data (discussed below).

Posterior probabilities are calculated in BBNs by using methods of belief updating based on Bayes' Theorem, where the posterior probability of species presence $S$, given habitat conditions $H$, is calculated as the product of the conditional probability of habitat given species presence and the marginal, $i$. e., unconditional probability, of species presence divided by the marginal probability of the habitat conditions, or:

$$
P(S \mid H)=\frac{P(H \mid S) P(S)}{P(H)}
$$

and the posterior probability of species absence 


$$
\bar{S}
$$

given habitat conditions is:

$$
P(\bar{S} \mid H)=\frac{P(H \mid \bar{S}) P(\bar{S})}{P(H)}
$$

For increasingly rare species and increasingly incomplete, i.e., uncertain and generalized, descriptions of habitat,

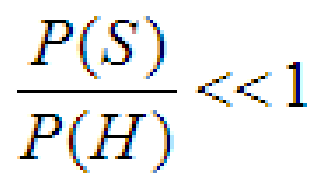

so that

\section{$\lim P(S \mid H) \rightarrow 0$}

and

\section{$\lim P(\bar{S} \mid H) \rightarrow 1$}

Further,

$$
P(S \mid H)+P(\bar{S} \mid H)=P(S \mid \bar{H})+P(\bar{S} \mid \bar{H})=1
$$

This means that false negatives, i.e., predicting species absence when it is actually present, and false positives, i.e., predicting species present when it is actually absent, can be greatly influenced by incorrectly parameterized conditional probabilities and prior marginal probabilities. This makes calibration, accuracy testing, and updating of the model with site data an important step in honing model accuracy.

There are many reasons for false negatives in apparently suitable habitat such as elusiveness or low detectability of the species, e.g., some hypogeous fungi produce aboveground fruiting bodies only once every few years, lack of training or experience of the observers, e.g., some rare bryophyte species grow sparsely and hidden among mats of more common species and are easy to miss, temporary absence in suboptimal habitat, and other conditions. False positives may be more difficult to determine but could include extinction debt, i.e., the organism is temporarily present after a disturbance stressor but is on its way out, occurrence in sink habitat that provides little contribution to population viability, incorrect identification, and other reasons. Thus, with very rare species, it was desirable to model and test rates of false negatives and false positives, that is, the accuracy of predicting presence and absence.

Further details on BBN structures and underlying statistical calculations can be found in Jensen , O'Hagan et al. (2004), Oliver and Smith (1990), and other references.

\section{Overall Bayesian belief network modeling process}

To develop BBN prediction models of rare species in the Pacific Northwest, I devised and followed a specific process (Fig. 1), which proved important to ensure that the models were rigorously structured, peer reviewed to reduce or avoid later conflicts among experts, and scientifically defensible. In this process, I served as what I will term here the "modeler," although in the literature on artificial intelligence programming and decision modeling the term "knowledge engineer" is also used in this same capacity (Fox 1984).

\section{Alpha-level model}

The first step is for the managers to prioritize species 
Fig. 1. Overall process of modeling rare species using Bayesian belief networks under the Northwest Forest Plan.
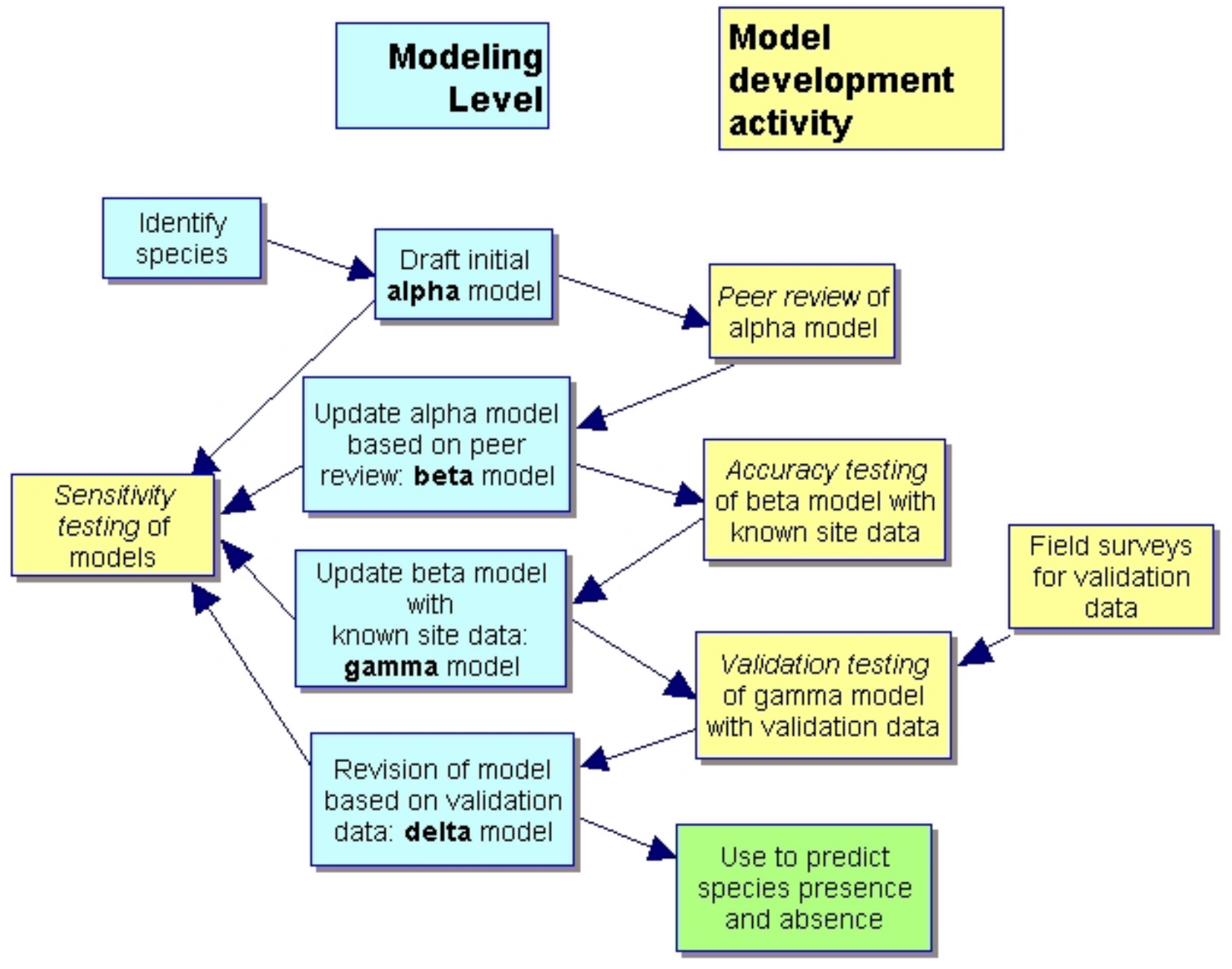

of management interest. The modeler then consults with a species expert(s) to develop the initial alphalevel model for a given species. In a meeting or panel setting, the expert first identifies key environmental correlates and stressors for the species, and the modeler represents this as an influence diagram representing the main environmental predictor variables and their links to the species response variable (Fig. 2). Then, with help and prompting of the modeler, the influence diagram is converted to a functional $\mathrm{BBN}$ by specifying appropriate states or equations and probabilities of each node in the diagram. In doing so, the species expert may consult the literature and their own data. What can be termed the "alpha-level model," a functional BBN, then represents the expert's knowledge and professional judgment on the major factors influencing the likelihood of presence of the species.

\section{Beta-level model}

In the next step, the alpha-level model is then subject to strict peer review overseen by the modeler. The alpha-level model is treated similar to a manuscript 
Fig. 2. A simple influence diagram showing key environmental correlates of presence of a rare fungus, Bridgeoporus nobilissimus (B. nob.). Best used as the basis for further modeling, the correlates should also express causal relations, so that the diagram becomes a "causal web." A. p. = Abies procera (noble fir), pres. $=$ present, $\mathrm{dbh}=$ diameter at breast height.

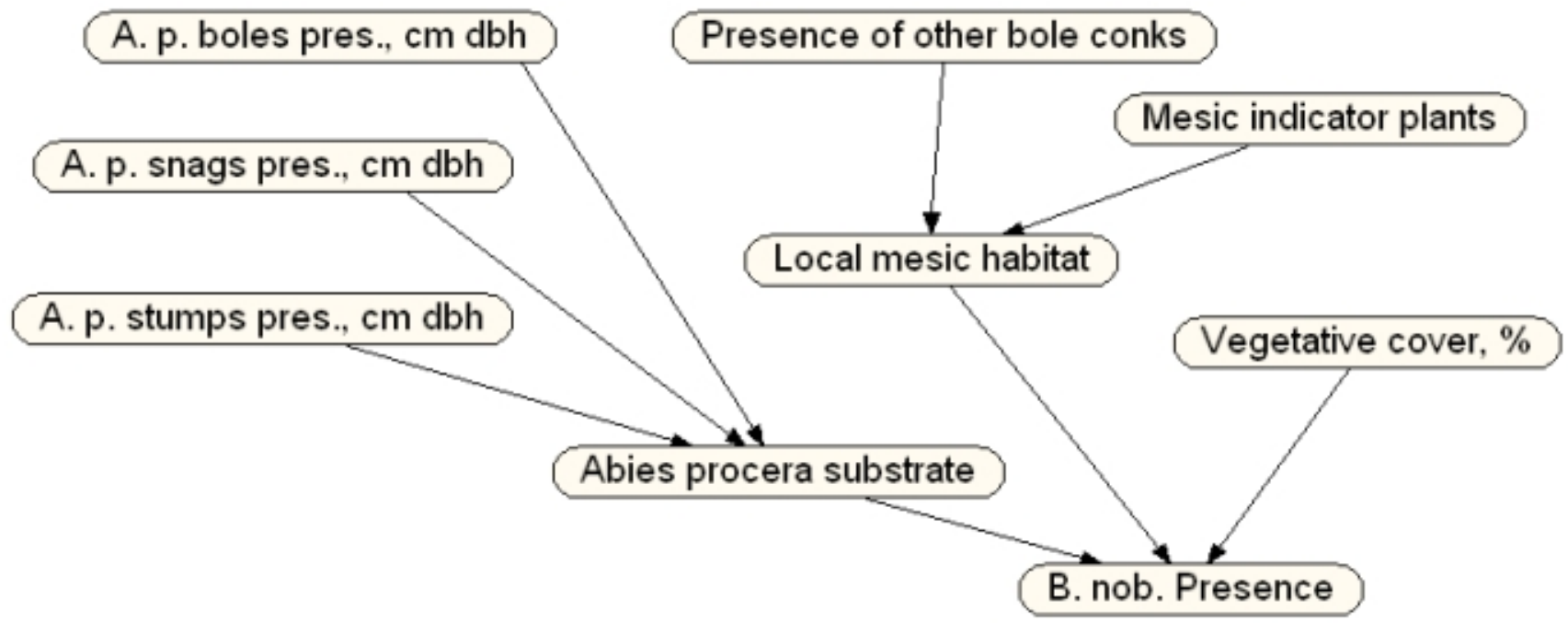

submitted to a journal, with the modeler serving as editor. Peer reviewers are selected as other experts on the species of interest, and they provide a formal critique on the alpha-level model's structure. As necessary, one or more competing model structures may be developed with the reviewers.

Next, the modeler presents the peer reviews to the initial expert who may choose to accept, modify, or reject the suggested changes. Rationales for the results are fully documented by the expert and the modeler. The model that the expert finalizes at this stage is the "beta-level model." If there is deviation in scientific opinion on the best model to use, multiple BBN models may be identified for the next stages.

\section{Gamma-level model}

The beta-level model(s) is then subject to accuracy testing with known site data on the species. Although the data may also have been consulted by the initial expert to help craft the alpha-level model, they were not used explicitly to create the model; at this stage, the data are more formally used to determine the degree to which the beta model(s) correctly predicts species presence and absence. Results of accuracy testing are presented in a confusion matrix.

For binary response variables such as species presence and absence, accuracy results also can be presented in a receiver operating characteristic (ROC) diagram, which plots percent correct predictions of species presence as a function of percent false predictions of species presence (Hand 1997). A ROC curve that hugs the upper left corner of the graph has best predictive power. The area under the curve, when plotted on normalized axes, is 1 when the model is error-free, 0.5 along the diagonal with fully random errors, and $<0.5$ below the diagonal when the model produces more errors than correct outcomes. The ROC curve also can be used to identify cutoff values matching desired thresholds of error rates. For example, if false negatives and false positives are equally undesirable, a cutoff on the ROC curve would correspond to the upper left corner of the graph. If 
false negatives are more undesirable the cutoff point would be more to the right on the graph, and if false positives are more desirable it would be more to the left. Thus, results of competing models can be plotted on the same ROC graph and their accuracy performance directly compared to each other and to desired performance criteria.

The known site data then can be used to update the prior and conditional probabilities in the beta-level model, or even the model's overall structure. Updating from case data is discussed below. This updated model is termed the "gamma-level model." The degree of bias in the known site data should be documented, if they were not based on random samples.

\section{Delta-level model}

Next, new validation data, if available, can be used to further test and update the gamma-level model, to produce a "delta-level model." This becomes the final version used to predict species presence and absence at the project level in the field. Validation data would be collected through statistically-based field sampling, where species presence and values of the predictor variables represented in the gamma level model are recorded from randomized plots or from plots on which the results of the model were being applied by the manager. In the case of very rare species, there may be no further validation data available, so the final model stage for use would be the previous gamma level model.

\section{Additional modeling guidelines}

More specific guidelines for creating and revising BBNs are presented by Marcot et al. (in press). These guidelines include ways to keep model structures simple so that conditional probabilities are easy to initially specify. Examples are specifying no more states per node than are necessary, keeping the number of parent nodes to four or fewer so as to keep conditional probability tables tractable, and building models broad rather than deep, that is, keeping the number of node layers to four or fewer as much as possible, so as not to unduly swamp out desired influences of input environmental parameters.

\section{Sensitivity testing}

Each stage in the model-building process uses sensitivity analysis in various ways to evaluate model structure and performance. In general, sensitivity analysis determines which inputs, i.e., habitat and stressor variables, most affect the specified response variables, and can be conducted on portions of a BBN as well as on the overall model.

During development of the alpha-level model, sensitivity analysis can help the species expert determine if the model is structured correctly, that is, according to their experience. During other stages in model development, it can be used to identify and prioritize the main environmental influences to help guide field validation studies. In the final model, it can help prioritize which stand or site attributes are most important for restoration activities.

With categorical states, as mostly used in the species-habitat BBNs, sensitivity is calculated in Netica (B. Boerlange, personal communication) as the degree of entropy reduction $I$, which is the expected difference in information bits $H$ between a variable $Q$ with $q$ states and a findings variable $F$ with $f$ states, and is calculated as:

$$
I=H(Q)-H(Q \mid F)=\sum_{q} \sum_{f} \frac{P(q, f) \log _{2}[P(q, f)]}{P(q) P(f)}
$$

Formulae used in sensitivity analysis of continuous variables (variance reduction) are presented by Marcot et al. (in press). In general, sensitivity analysis quantifies the degree $I$ to which an input variable $Q$ influences the response variable $F$ within the BBN network. The greater the value $I$, the greater the influence.

\section{Testing model performance and updating model structure with case data}

One of the major advantages of the BBN approach is use of case data to help update model probabilities or model structure. This nicely combines expert knowledge with empirical data (Heckerman et al. 1994). Updating is done using Bayesian learning and the specific method used is called the 
expectation maximization (EM) algorithm (Appendix 1; Watanabe and Yamaguchi 2003). With an adequate set of case data, the EM algorithm essentially compares outcomes predicted by the model with outcomes from real-world observations, and incrementally adjusts conditional probabilities of the intermediate nodes to better fit the cases.

The EM algorithm also adjusts the prior probabilities of input variables according to the frequencies of the states of each input node in the case data. With no prior information, I had set all probabilities of input nodes to uniform distributions, which represent complete uncertainty. The case data then helped adjust these probabilities to more realistic frequencies based on conditions observed in the field. The overall result of applying the EM algorithm with case data is a model having updated prior and conditional probabilities, that better fits actual site data, and that presumably better predicts species presence or absence from unknown site conditions. Advantages over a more traditional statistical modeling method is that the EM algorithm for updating probability tables, and the testing of BBN model prediction accuracy, can both function with missing data and can apply user-specified weights of the case data.

\section{RESULTS}

Results will be presented here using the example Bayesian belief network (BBN) model of the rare fungus discussed above.

\section{Alpha-level model: expert experience}

Initial consultation with the species experts resulted in developing a first-round influence diagram and alpha-level model based on their personal experience and expectations of the key environmental correlates that determine presence or absence of the species. The alpha-level model, not shown here, included the same variables, but different conditional probabilities, as ended up in the beta-level model (Fig. 3a), except for node B. In the alpha-level model, this node was specified as "distance to wet place," with three distance ranges specified (0-10 m,> 10-100 m, and > $100 \mathrm{~m}$ ). This node was designed to determine the proximity to forested wetlands, including seeps, as initial experience of the experts suggested that such microenvironments might at least correlate with presence of the fungus species of interest.

\section{Beta-level model: peer review}

In the beta-level model, it was determined through peer review and reconciliation with the original experts that node $\mathrm{B}$ was better expressed as "presence of other bole conks" as more direct indicators of potential adequacy of bole wood decay and suitable microenvironment for inoculation of this species. I will use this revised node as an example of how each variable in each modeling stage was carefully specified and defined by the experts.

This revised node was defined specifically as presence of one or more other fungi species including Ganoderma spp. and Fomitopsis spp., but that some other bole conks, particularly Phellinus pini should be ignored because they do not serve as such indicators for the species of interest. The reviewers and initial experts noted that presence of Ganoderma is particularly interesting and potentially an important indicator, and that this correlation could be treated as a working hypothesis to be empirically tested.

The indicator conks might occur on noble fir (Abies procera) or on other host tree species such as western hemlock (Tsuga heterophylla), Douglas-fir (Pseudotsuga menziesii), and others, of any size. The two states specified for this node were rare to absent, and present. "Rare to absent" was defined as $<1 / 3$ of suitable tree snags and stumps in the stand having a conk of the indicator genera noted above, where suitable means that the tree is not freshly dead or is at least of snag decay class 3 on a 5-class decay scale. "Present" was defined as the same but with $\geq 1 / 3$ of the trees with such indicator conks. It was further noted in the model documentation that absence of indicator conks may occur from case hardening of the trees from fire charring caused by hot burns, i.e., either prescribed or wild fires, which also creates unsuitable conditions of the species in question, so this indicator pertains to post-fire forest environments as well.

Note that the definitions and specifications of this node, like those of each input node, clearly explain what an observer should measure in the field when determining the correct state or value for the node. 
Fig. 3. Two stages (see Fig. 1) in the modeling of a rare fungus using Bayesian belief networks as developed from the influence diagram shown in Fig. 2. (a) beta-level model after peer review, (b) gamma-level model after incorporating known site field data (Table 1). See text for discussion of the initial alpha-level model. No delta-level model is available as further field validation studies had not been conducted.

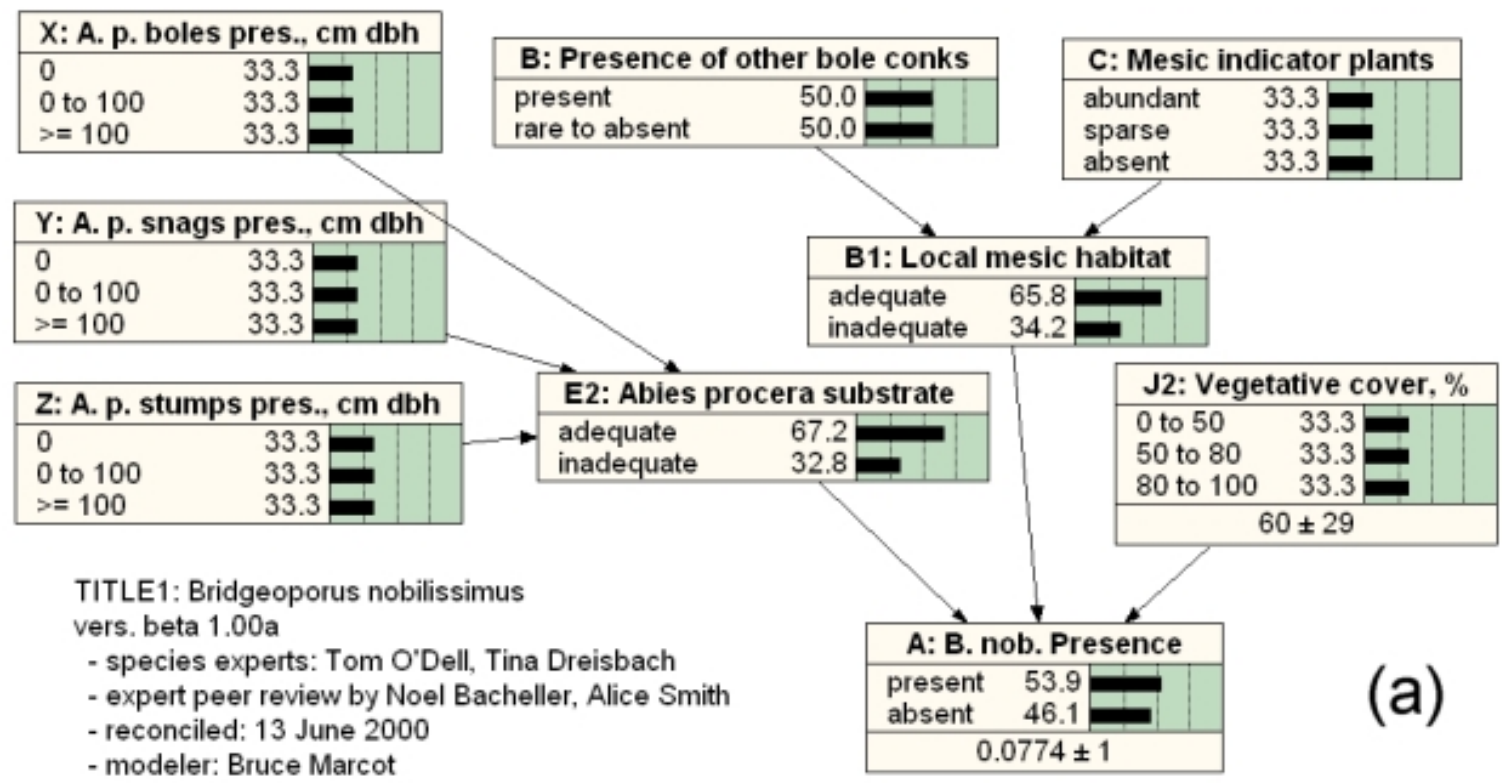

\begin{tabular}{|c|c|}
\hline \multicolumn{2}{|c|}{ X: A. p. boles pres., cm dbh } \\
\hline $\begin{array}{l}0 \\
0 \text { to } 100 \\
>=100\end{array}$ & $\begin{array}{l}18.3= \\
30.8 \\
50.9\end{array}$ \\
\hline
\end{tabular}

\begin{tabular}{|ll|}
\hline \multicolumn{3}{|c|}{ Y: A. p. snags pres., cm dbh } \\
\hline 0 & 57.0 \\
0 to 100 & 23.6 \\
$>=100$ & 19.4 \\
\hline
\end{tabular}

\begin{tabular}{|l|l|}
\hline Z: A. p. stumps pres., cm dbh \\
\hline 0 & 22.8 \\
0 to 100 & 11.5 \\
$>=100$ & 65.7 \\
\hline
\end{tabular}

TITLE1: Bridgeoporus nobilissimus vers. gamma $1.00 \mathrm{a}$

- species experts: Tom O'Dell, Tina Dreisbach

- expert peer review by Noel Bacheller, Alice Smith

- reconciled: 13 June 2000

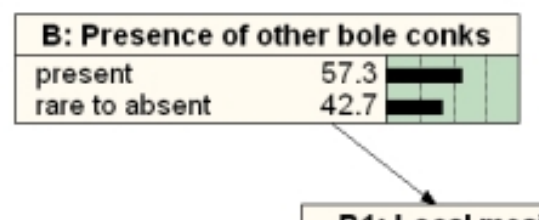

\begin{tabular}{|l|l|}
\hline \multicolumn{2}{|c|}{ C: Mesic indicator plants } \\
\hline abundant & 59.4 \\
sparse & 33.3 \\
absent & 7.25 \\
\hline
\end{tabular}

- modeler: Bruce Marcot

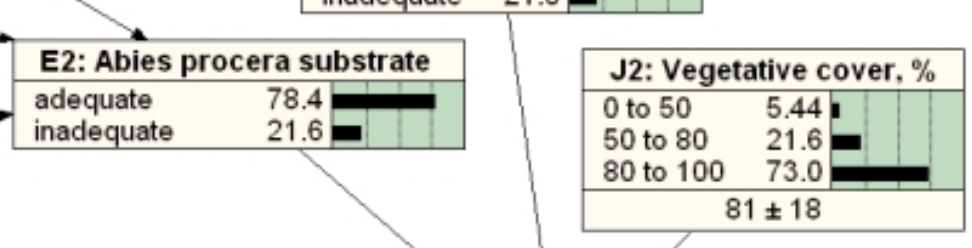

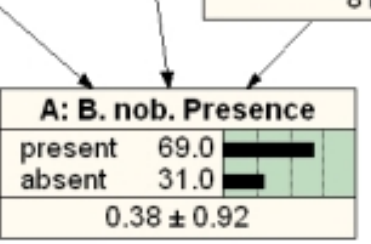

(b) 
Such documentation is extremely important to ensuring that the model is run and validation data are correctly collected as intended by the experts.

\section{Gamma-level model: accuracy testing and updating with known site data}

The beta-level model was first tested for accuracy in predicting presence and absence of the species by using a set of known site data (Table 1) provided by the original species experts. The resulting confusion matrix (Table 2) showed that, out of 31 known cases of species presence, the beta model correctly predicted presence as the more likely outcome in all cases, but out of 14 known cases of species absence the beta model correctly predicted absence in only $3(21 \%)$ of the cases. This meant a $0 \%$ error rate of predicting presence, a $79 \%$ error rate in predicting absence, and an overall error rate of $24 \%$, with this data set. Management implications of the error rates are discussed below. I then plotted the error rates on a ROC curve (Fig. 4), which approaches the upper left corner of the graph, suggesting overall a fairly accurate predictive model.

I updated the beta model with the case file data (Table 1) using the expectation maximization (EM) algorithm. This changed the probability tables of the model to better conform to the case data, as shown in the comparison of the beta model (Fig. 3a) and the gamma model (Fig. 3b). The prior probabilities of the input nodes (nodes B, C, J2, X, $\mathrm{Y}$, and $\mathrm{Z}$ in Fig. 3) were adjusted by the EM algorithm from uniform, i.e., complete uncertainty or complete lack of prior data, to the observed frequencies of the states for each node. For example, in node $\mathrm{J} 2$, the frequency of dense (80-100\%) vegetative cover changed from $33 \%$ in the beta model before incorporating the case data, to $73 \%$ after. Adjusting prior probabilities to known frequencies of states is an important step in refining the accuracy of the model.

Incorporating case data also served to update the CPTs of the other nodes in the model. For example, the probabilities in the outcome node of species presence or absence (node A in Fig. 3) changed most dramatically for some conditions such as for Abies procera substrate being adequate, vegetative cover being $80-100 \%$, and local mesic habitat being inadequate; in this example, the probability of species presence dropped from $80 \%$ in the beta model to about $43 \%$ in the gamma model after incorporating the case file (Table 3 ). This change in the CPT better matched the known field conditions in the case data.

The changes in prior and conditional probabilities from incorporating the case data slightly increased the performance accuracy of the gamma model to about $22 \%$ total error rate, down from $24 \%$ in the beta model, because one additional case of known absence was now correctly predicted as absence. The gamma model did not perform perfectly, likely because there are still unknown predictive variables not included in the model.

Incorporating the case data changed the relative sensitivity structure of the model rather dramatically (Fig. 5). In the beta model, three input variables nearly equally most affected prediction of species presence and absence: percent vegetative cover, Abies procera stumps, and A. procera snags. After incorporating the case data, $A$. procera stumps became by far the most influential variable, followed weakly by presence of other bole conks and percent vegetative cover. In both models, $A$. procera live boles and presence of mesic site plant indicators had very weak influence, and could likely be dropped from the model, i.e., the nodes can be "absorbed" into the network structure retaining their influence in the remaining variables, and from future validation data collection.

\section{DISCUSSION}

\section{Efficacy of building Bayesian belief network species-habitat models}

Building Bayesian belief network (BBN) habitat models of rare species can be done in a very efficient way, using procedures of knowledge engineering to solicit expert information. Most species experts were easy to work with and interested in codifying their knowledge into working models for further review, testing, and refinement. A few experts, as to be expected, balked at the task because they viewed models as definitive quantitative tools that should reflect near-perfect understanding of species' ecologies. This, of course, is not at all the intent of the modeling done here, which instead is aimed at organizing thinking and structuring of current knowledge and expertise for further testing. 
Fig. 4. A receiver operating characteristic (ROC) curve showing results of testing the classification success of the beta-level model in Fig. 3a with the case file data in Table 1. A ROC curve plots percent true positives, sensitivity, against percent false positives, 1-specificity. See text for further explanation.

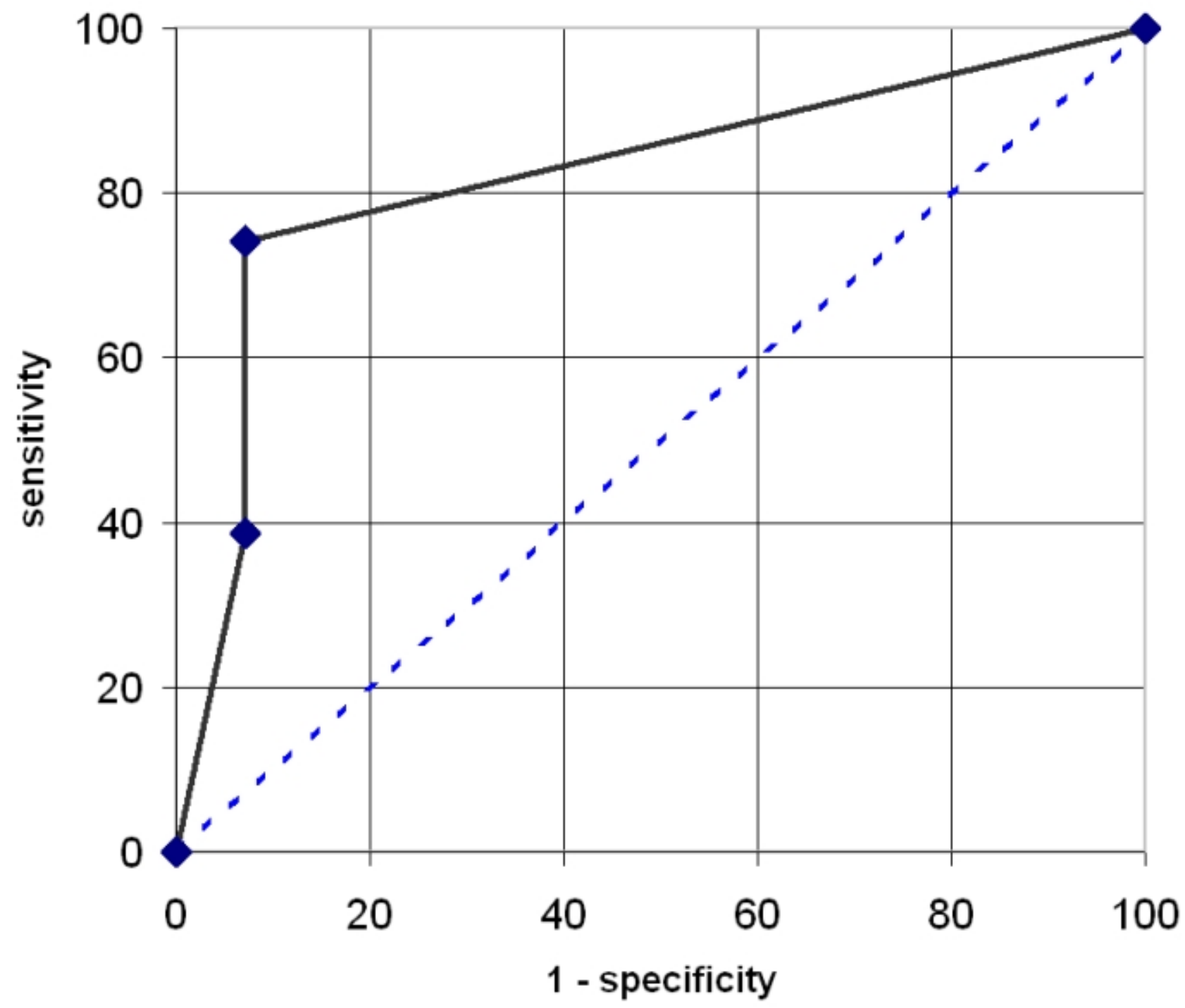

The BBN modeling construct serves as an intuitive picture of what experts know about species-habitat relations. Together with the simpler influence diagrams, demonstrating a working $\mathrm{BBN}$ to managers is a very effective communication tool to help them discern the kinds of sites where rare species may occur and the specific site conditions that might be targeted for conservation or restoration of the species. 
Table 1. Example of a case data table of a species from multiple surveys. Such data can be used to induce an initial network structure or to refine probabilities of relations in an existing network. Column headings refer to case number, and input and output nodes in the model for the rare fungus Bridgeoporus nobilissimus (B. nob.; Fig. 3a). * = missing data. (Data sources: T. O'Dell and T. Dreisbach, personal communication).

\begin{tabular}{|c|c|c|c|c|c|c|c|}
\hline \multirow[b]{2}{*}{ Case number } & \multicolumn{6}{|c|}{ Input nodes } & \multirow{2}{*}{$\begin{array}{c}\text { Output node } \\
\text { Node A: } B . \\
\text { nob. presence }\end{array}$} \\
\hline & $\begin{array}{c}\text { Node X: } \\
\text { Abies procera } \\
\text { boles, cm dbh }\end{array}$ & $\begin{array}{c}\text { Node Y: } \\
\text { Abies procera } \\
\text { snags, cm dbh }\end{array}$ & $\begin{array}{c}\text { Node Z: } \\
\text { Abies procera } \\
\text { stumps, cm } \\
\text { dbh }\end{array}$ & $\begin{array}{l}\text { Node B: } \\
\text { Other bold } \\
\text { conks }\end{array}$ & $\begin{array}{l}\text { Node C: } \\
\text { Mesic indicator } \\
\text { plants }\end{array}$ & $\begin{array}{l}\text { Node J2: } \\
\text { Vegetative } \\
\text { cover, } \%\end{array}$ & \\
\hline 1 & 0 & 0 & 130 & rare to absent & sparse & 85 & present \\
\hline 2 & 0 & 0 & 110 & present & abundant & 85 & present \\
\hline 3 & 0 & 0 & 130 & present & abundant & 80 & present \\
\hline 4 & 100 & 100 & 0 & present & abundant & 85 & present \\
\hline 5 & 90 & 0 & 100 & present & abundant & 45 & absent \\
\hline 6 & $*$ & $*$ & $*$ & $*$ & abundant & 95 & absent \\
\hline 7 & 100 & $*$ & $*$ & $*$ & abundant & 100 & absent \\
\hline 8 & 75 & $*$ & $*$ & $*$ & sparse & 100 & absent \\
\hline 9 & 75 & 50 & 75 & $*$ & sparse & 100 & absent \\
\hline 10 & 75 & $*$ & $*$ & $*$ & absent & 70 & absent \\
\hline 11 & 150 & 150 & 150 & $*$ & abundant & 100 & absent \\
\hline 12 & 150 & $*$ & $*$ & $*$ & sparse & 100 & absent \\
\hline 13 & 150 & $*$ & $*$ & $*$ & abundant & 100 & absent \\
\hline 14 & 100 & 100 & 100 & $*$ & absent & $*$ & absent \\
\hline 15 & 75 & 50 & $*$ & $*$ & abundant & 100 & absent \\
\hline 16 & 100 & 0 & 0 & $*$ & absent & 80 & absent \\
\hline 17 & 75 & 75 & 75 & $*$ & sparse & 50 & absent \\
\hline 18 & 80 & 50 & 50 & $*$ & abundant & 100 & absent \\
\hline 19 & $*$ & $*$ & $*$ & $*$ & abundant & 80 & present \\
\hline 20 & $*$ & $*$ & 107 & $*$ & abundant & 100 & present \\
\hline 21 & $*$ & $*$ & $*$ & $*$ & abundant & 100 & present \\
\hline 22 & $*$ & $*$ & 122 & $*$ & abundant & $*$ & present \\
\hline 23 & $*$ & $*$ & $*$ & $*$ & abundant & $*$ & present \\
\hline
\end{tabular}




\begin{tabular}{|c|c|c|c|c|c|c|c|}
\hline 24 & 50 & 100 & 0 & present & sparse & 90 & present \\
\hline 25 & 100 & 100 & 0 & present & abundant & 75 & present \\
\hline 26 & 0 & 0 & 100 & rare to absent & sparse & 75 & present \\
\hline 27 & 100 & 0 & 100 & present & sparse & 70 & present \\
\hline 28 & 100 & 0 & 100 & present & abundant & 90 & present \\
\hline 29 & 0 & 0 & 100 & rare to absent & abundant & 93 & present \\
\hline 30 & 100 & 100 & 0 & rare to absent & abundant & 85 & present \\
\hline 31 & 0 & 0 & 100 & rare to absent & abundant & 95 & present \\
\hline 32 & 100 & 0 & 100 & rare to absent & sparse & 70 & present \\
\hline 33 & 100 & 100 & $*$ & present & sparse & 90 & present \\
\hline 34 & 100 & 0 & 100 & present & sparse & 90 & present \\
\hline 35 & 0 & 0 & 100 & present & abundant & 95 & present \\
\hline 36 & 100 & 0 & 100 & rare to absent & abundant & 95 & present \\
\hline 37 & 100 & 30 & 0 & rare to absent & abundant & 80 & present \\
\hline 38 & 30 & 30 & 100 & present & abundant & 80 & present \\
\hline 39 & 30 & 0 & 100 & present & sparse & 44 & present \\
\hline 40 & 100 & 10 & 100 & rare to absent & sparse & 85 & present \\
\hline 41 & 100 & 0 & 100 & present & sparse & 95 & present \\
\hline 42 & 100 & 30 & 100 & present & abundant & 85 & present \\
\hline 43 & 10 & 0 & 100 & present & abundant & 75 & present \\
\hline 44 & 30 & 0 & 100 & rare to absent & abundant & 70 & present \\
\hline 45 & 100 & 0 & 100 & present & sparse & 58 & present \\
\hline
\end{tabular}

\section{Model reliability and utility}

Some 12 rare species were modeled using BBNs as demonstrated in this paper, to varying points along the modeling path (Fig. 1). Models still in alpha level should not be used with much confidence, whereas gamma and especially delta-level models would be released for general use to help with management decisions. Although they may have great utility and fulfill a need, the models even at these later stages of refinement and updating still carry some degree of unreliability because they deal with prediction of rare events, which is always wrought with uncertainty.

Some of the uncertainties that these simple models do not directly address are: (1) differential detectability of species given specific survey protocols, although detectability can be incorporated into the BBN model structure, e.g., see the BBN model predicting capture of northern flying squirrels, Glaucomys sabrinus, presented in Marcot et al., in press; (2) measurement error in defining the input variables and species identification; (3) 
Table 2. Example of a confusion matrix showing the prediction accuracy of the Bayesian belief network (BBN) beta model in Fig. 3a used with the case data file in Table 1. The confusion matrix shows the number of known cases that were correctly classified, here as whether the species was actually present or absent, given the conditions on each site.

\begin{tabular}{ccc}
\hline \hline & Predicted & \\
\hline Present & Absent & Actual \\
31 & 0 & Present \\
11 & 3 & Absent
\end{tabular}

uncertainty in how input variables are gathered into intermediate, i.e., latent variables; and (4) propagation of error of covariates throughout the model structure. Also missing are direct representations of potential reasons for false negatives and false positives, as listed above, such as dynamics of metapopulations and source-sink habitat use.

Although such errors and modeling lapses may be egregious in models intended to explain biological systems, they were tolerable in these models intended to guide priorities for species surveys. The models did represent uncertainty in stressors and environmental conditions through prior probabilities of the input variables, and in combinations of such conditions through conditional probabilities of the other model variables. The models represented outcomes as probabilities, which fit well in a risk analysis and risk management framework. The models also were fully documented, especially including peer reviews and reconciliations, which greatly helped reduce concerns for creating biased models representing just one expert's belief system.

\section{Implications of model predictions and use in management}

It was imperative to make the potential model users, e.g., the resource managers and decision makers, understand that such models should not be used to definitively dictate management activities based on model predictions of rare species presence or absence. The model outcome should not be the decision. Rather, the stated purpose was to help guide management decisions on conducting expensive and intensive species surveys, or on at least temporarily forgoing site-disturbing activities until such surveys could be done, by identifying sites with higher likelihoods of rare species presence.

Type I and II errors, i.e., false negatives and false positives, have very different implications for rare species management. False negatives meant that the models would predict species absence when it was indeed present. This could mean that site-disturbing activities might proceed and could result in local extirpation of the species. There would be no specific economic cost directly associated with this unknown model outcome; the cost instead would be to local persistence of the species. On the other hand, false positives meant that the models would predict species presence when it was indeed absent. This could mean that site-disturbing activities would be postponed or halted, thus possibly incurring a high opportunity cost such as forgoing timber harvest. It could also mean expenditure of time and money to conduct expensive species surveys where the species does not exist. Thus, there would be very real short-term economic implications associated with false positives, but no real harm to the species.

The example model presented in this paper accurately predicted species presence, but incurred a fairly high error rate in predicting species absence, that is, false positives. Thus, the manager might use this model with some caution when forestalling sitedisturbing management activities and directing species surveys, and, depending on cost, may wish 
Fig. 5. Results of sensitivity analyses of the beta- and gamma-level models (Fig. 3). Bars represent the influence analyzed as entropy reduction. See text for calculation of each predictor variable on predicted presence or absence of the species; greater bar heights denote greater influence.

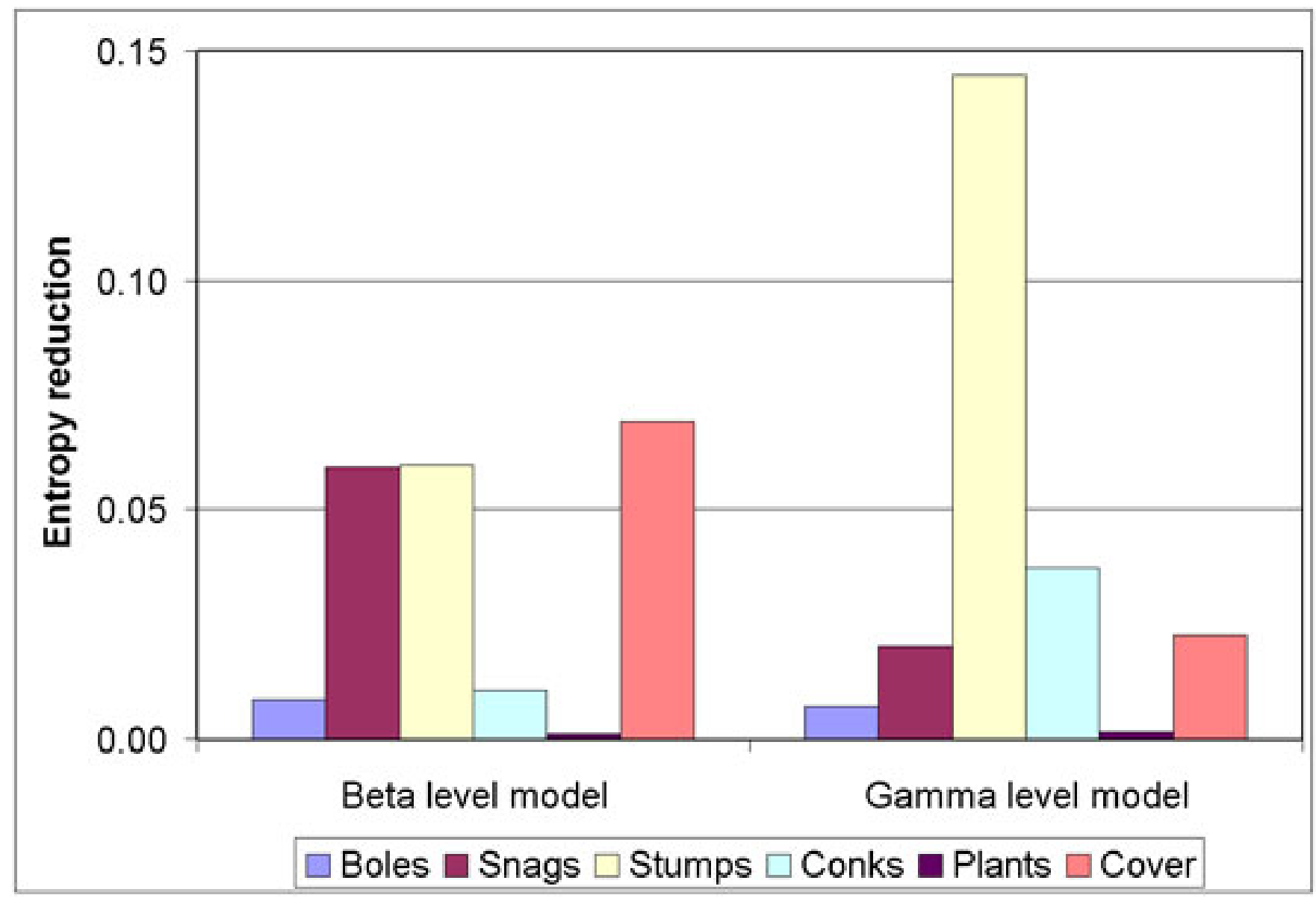

for a "second opinion" from specialists for a site inspection when the model predicts species presence, at least in some marginal situations. On the other hand, in using this particular model, the manager would have far less concern over inadvertently doing local harm to the species. There is greater assurance of accuracy when results of this model would suggest species absence, implying not conducting species surveys, and not forgoing sitedisturbing activities. Also, false positives could be interpreted a different way. Areas with false positives located adjacent to known locations, i.e., true positives, could be considered good potential habitat for conservation.
Other species models may be more balanced in error structure or may be tipped the other way. Thus, the modeler should inform the manager as to the error structure of the model and its potential implications. The manager would then assert their risk attitude toward appropriate interpretations and use of each model.

\section{Using the model for site conservation and restoration}

The manager could use sensitivity results of the model to prioritize which site attributes or stand conditions to conserve or restore to help provide for 
the species. For example, the sensitivity analysis outcome of the gamma-level model (Fig. 5) would suggest that retaining large $(\geq 100 \mathrm{~cm}$ diameter) legacy stumps and snags of Abies procera in stands with other bole conks as specified above, and fostering moderately dense $(50-80 \%)$ cover of tall shrubs or trees, i.e., the more optimal condition for this species, could be useful to provide suitable habitat for this fungus species. Such conservation or restoration activities could be treated as testable management hypotheses and response of the species could be studied in the field. Data could be compiled into a validation database and, in the spirit of adaptive learning, used to further test and refine the model.

As multiple models become available on other rare species, it may be possible to link them with common stand attributes. In this way, multispecies predictions could be made with common databases on site conditions.

\section{CONCLUSIONS}

Bayesian belief models (BBN) of rare specieshabitat relationships are relatively easy to build and can serve well to represent experts' experience. They also are solid tools for combining empirical data with expert judgment, and for helping to organize current knowledge and to communicate with and advise habitat managers.

It is important that the BBN model-building process include peer review, reconciliation, and testing and updating with unbiased, known site data on the species of interest. Although the error rates of false negative and false positive model predictions of species presence and absence might not be greatly altered by incorporating some case data, model sensitivity, and even model structure, e.g., inclusion of exclusion of nodes, the states represented in nodes, and how the nodes are linked, can change a great deal with new data. This has implications for using such models to guide activities including prioritizing sites for species surveys, prioritizing stand conditions for habitat conservation or restoration, and revealing gaps in knowledge.
Responses to this article can be read online at: http://www.ecologyandsociety.org/voll1/iss2/art10/responses/

\section{Acknowledgments:}

My deep appreciation to mycologists Thom O'Dell and Tina Dreisbach who provided their expertise to help build the example model, provided the case data, and reviewed the manuscript. Noel Bacheller and Alice Smith served as peer reviewers of the model. Thanks also to Randy Molina, Marianne Turley, and two anonymous reviewers for comments on the manuscript. Mention of commercial products does not necessarily constitute endorsement by USDA Forest Service. This work was done under auspices of Pacific Northwest Research Station and Pacific Northwest Regional Office, USDA Forest Service.

\section{LITERATURE CITED}

Burdsall, H. H., Jr., T. J. Volk, and J. F. Ammirati, Jr. 1996. Bridgeoporus, a new genus to accommodate Oxyporus nobilissimus (Basidiomycota, Polyporaceae). Mycotaxon 60:387-395.

Castillo, E., J. M. Gutierrez, and A.S. Hadi. 1997. Expert systems and probabilistic network models. Springer-Verlag, New York, New York, USA.

Dempster, A., N. Laird, and D. Rubin. 1977. Maximum likelihood from incomplete data via the EM algorithm. Journal of the Royal Statistical Society 39(Series B):1-38.

Fox, J. 1984. A short account of knowledge engineering. The Knowledge Engineering Review 1 (1):4-14.

Hand, D. 1997. Construction and assessment of classification rules. John Wiley and Sons, New York, New York, USA.

Heckerman, D., D. Geiger, and D. M. Chickering. 1994. Learning Bayesian networks: the combination of knowledge and statistical data. Pages 293-301 in R. L. de Mantaras and D. Poole, editors. Uncertainty in artificial intelligence. Proceedings of the Tenth Conference. Morgan Kaufmann, San Francisco, University of Washington, Seattle, California, 
USA.

Jensen, F. V. 1996. An introduction to Bayesian networks. UCL Press, London, UK.

Lee, D. C. 2000. Assessing land-use impacts on bull trout using Bayesian belief networks. Pages 127147 in S. Ferson and M. Burgman, editors. Quantitative methods for conservation biology. Springer, New York, New York, USA.

Lehmkuhl, J. F., J. G. Kie, L. C. Bender, G. Servheen, and H. Nyberg. 2001. Evaluating the effects of ecosystem management alternatives on elk, mule deer, and white-tailed deer in the interior Columbia River Basin, USA. Forest Ecology and Management 153(1-3):89-104.

Lesher, R. D. 2005. An environmental gradient model predicts the spatial distribution of potential habitat for Hypogymnia duplicata in the Cascade Mountains of northwestern Washington. Dissertation. University of Washington, Washington, USA.

Marcot, B. G., P. A. Hohenlohe, S. Morey, R. Holmes, R. Molina, M. Turley, M. Huff, and J. Laurence. 2006. Characterizing species at risk II: using Bayesian belief networks as decision support tools to determine species conservation categories under the Northwest Forest Plan. Ecology and Society, in press.

Marcot, B. G., R. S. Holthausen, M. G. Raphael, M. M. Rowland, and M. J. Wisdom. 2001. Using Bayesian belief networks to evaluate fish and wildlife population viability under land management alternatives from an environmental impact statement. Forest Ecology and Management 153 (1-3):29-42.

Marcot, B. G., J. D. Steventon, G. D. Sutherland, and R. K. McCann. 2006. Guidelines for developing and updating Bayesian belief networks for ecological modeling. Canadian Journal of Forest Research, in press.

Marin, J. M., R. Montes-Diez, and D. Rios-Insua. 2003. Bayesian methods in plant conservation biology. Biological Conservation 113(3):379-387.

O'Hagan, A., J. Forster, M. G. Kendall, A. Stuart, and J. K. Ord. 2004. Kendall's advanced theory of statistics: Bayesian inference. Second edition. Arnold, London, UK.
Oliver, R. M., and J. Q. Smith, editors. 1990. Influence diagrams, belief nets and decision analysis. John Wiley and Sons, Chichester, UK.

Raphael, M. G., M. J. Wisdom, M. M. Rowland, R. S. Holthausen, B. C. Wales, B. G. Marcot, and T. D. Rich. 2001. Status and trends of habitats of terrestrial vertebrates in relation to land management in the interior Columbia River Basin. Forest Ecology and Management 153(1-3):63-87.

Rowland, M. M., M. J. Wisdom, D. H. Johnson, B. C. Wales, J. P. Copeland, and F. B. Edelmann. 2003. Evaluation of landscape models for wolverines in the interior northwest, United States of America. Journal of Mammology 84(1):92-105.

Schnute, J. T., A. Cass, and L. J. Richards. 2000. A Bayesian decision analysis to set escapement goals for Fraser River sockeye salmon (Oncorhynchus nerka.) Canadian Journal of Fisheries and Aquatic Sciences 57:962-979.

Spiegelhalter, D. J., A. P. Dawid, S. L. Lauritzen, and R. G. Cowell. 1993. Bayesian analysis in expert systems. Statistical Science 8(3):219-283.

U.S. Department of Agriculture Forest Service, and U.S. Department of the Interior Bureau of Land Management (USDA and USDI). 1994. Record of decision for amendments to Forest Service and Bureau of Land Management planning documents within the range of the northern spotted owl. Standards and guidelines for management of habitat for late-successional and old-growth forest related species within the range of the northern spotted owl. Washington, D.C., USA.

Watanabe, M., and K. Yamaguchi, editors. 2003. The EM algorithm and related statistical models. Marcel Dekker, New York, New York, USA.

Weisstein, E. W. 2005. Dirichlet function. From MathWorld-A Wolfram Web Resource. Available online at: http://mathworld.wolfram.com/DirichletF unction.html. 
Appendix 1. Overview of the expectation maximization (EM) algorithm used to update conditional probabilities.

Please click here to download file 'appendix1.pdf'. 